\title{
Reorganization and Innovation -- Analysis of Singing Skills of'New Voice'
}

\section{Works}

\section{YangWei}

\author{
Dingxi Teachers College, Gansu, China
}

\begin{abstract}
Key words: new voice, new music, reorganization and innovation
\end{abstract}
\begin{abstract}
About the 'new voice' concept, it should be said that its emergence and development already is the conventional result of singing form, however, the presentation of the 'reorganization and innovation' of modern 'new voice' in specific work is the key point of this field for our research. In this paper, it inherits some features of the 'new voice' works, furthermore explores and summarizes the relevant 'new voice' works, besides, goes about studying the 'new' points of 'voice' singing and the the innovative attempt of vocalization concept, apart from that, according to human voice's instrumental property, it takes analysis of the innovative variation and special treatment and destroys the limitation of concreteness and semantic feature of previous human voice singing. Besides, it extends to that people have felt that the built superficial rational order, value system, even the general beauty-appreciation of cultural spirit thousands of years ago is experiencing quietly super exceptional changing. 'Foreign things serving China' 'view the world by standing on the shoulder of giant' may be the traditional method most worthy of borrowing, which just like the product of multi-thinking and the 'mosaic' concept of post-modern music is also the demonstration of multi-culture development, which market prospect cannot be ignored and it inevitably will be the innovation, breakthrough, fusion of the new music, besides, will go forward along with the musical wave and be the best result of globalization. The author is not good enough in knowledge and talent, so for the deficiencies in this paper, pleasant to learn from others.

About the specific application of 'new voice', we still have lots of examples to prove its innovation and fusion and it is almost the new-type reorganization and innovation that creates the new voice concept and adds light and extraordinary splendour to the works. Here, the author will take technical analysis and context-semantics analysis of 'new voice' works singing.
\end{abstract}

\section{Technical Treatment on the Voice of Singer}

In terms of vocalizing skills, it is difficult for us to master the timbre unification of voice change area, for past traditional vocal music works, in order to make the timbre unified and smooth, we have spent great effort to do the 'trace removal work'. For the 'new voice' works, the mix of real tone and falsetto has stepped over the general conventional timbre unification concept and now it is serving the works emotion in high level and with high requirement, so that to give the nature of sound and emotion back. Phantom(Banshee)--Elegy and Vocalise for Soprano and Sound Piano is George Crumb's first English vocal work and the first(debut) work for human voice and piano. The special use of background piano sound amplification technology has magically described the shadow overhead feeling of Phantom. When singing, the singer pay attention to the accurate expression of emotion, which is surprising sometimes, pleasant sometimes, contemplative sometimes and sad sometimes. For the text part, it is taken from the When Lilacs Bloom in the Courtyard of Recollections of Lincoln(Wouter Whiteman Poetry) and it mainly adopts the natural 
twittering, furthermore, the imaginative description of death is expressed incisively and vividly. Actually, for the presentation state of sound, there is large number of technical content besides, the alternative use of real tone and falsetto, the mutual exchanging of cavity inside and outside etc. cannot be mastered immediately by anybody anytime.

Music score example: Phantom(Banshee)--Elegy and Vocalise for Soprano and Sound Piano Written by George Crumb

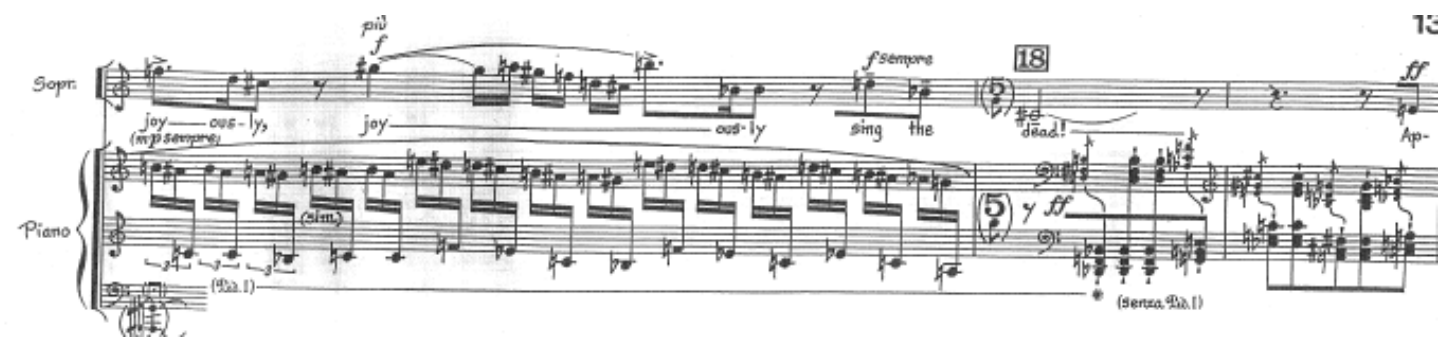

For the modern works, the vocal range of'new voice' music in performance can be broad according to the work demand, besides, it will not be limited by one certain voice register and selects timbre and sound, in which there is the accumulated sounding of singing, smiling, whispering and interjection, along with the emotional changes. Mostly, there is one certain range of pitch, which puts forward higher technical requirement and voice master ability for singers. The vocabulary 'North wind' appears in the Imitating of Long Ancient Autumn Night in Song of the Earth written by Ye Xiaogang, when in the performance, it requires the pianissimo treatment with continuous beat 1,3 of high D. All the singers know that for the vocalizing skills, it is easy to sing the high pitch but difficult to sing bass, furthermore, there is such long time of duration in this voice register, therefore, it needs the singer to use better the vocal cords closing, breath support and keep of voice flowing to leave audience imaginary sphere.

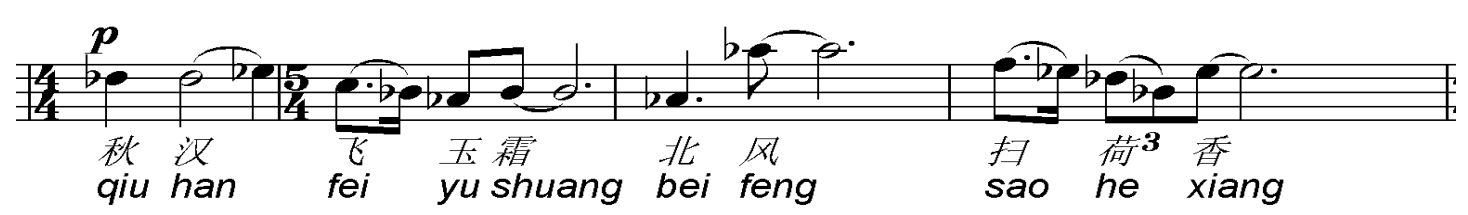

Music score example: Imitating of Long Ancient Autumn Night in Song of the Earth written by Ye Xiaogang

The people learning vocal music all know that, it is difficult to sing many sounds with one simple tone. For many individual high pitch, how to sing it energetically but not stiffly can test one singer's singing ability, besides, for the 'new voice' works, the expression of 'simple tone' has already become the common method. The light or heavy, strong or weak, high or low, rise or fall, fast or slow, urgent or gently, pause or transition, continuous or disconnected, tone or musical language, loose and tight management arrangement of vocal music all can be represented by the volume strength changes, besides, they can display the internal vigor of notes according to the natural demand of works' emotion expression. The famous American musical analyst and musical critic Mary Vinastrom keeps the opinion that the key point playing decisive function on the final shaping of one musical composition and getting more notes accumulate and release energy is the parameter of pitch, timbre, strength and value, all of which can form the musical form function without limitation in the process of music operation, like the post-modern music Mental State written by Stockhausen, Piano Phase written by Raich, etc. The phenomenon often appear in the 
'new voice' works and after singers' strength master and processing of the voice, the works will be presented with imaginative elements.

Music score example: Sequenza III for Voice written by Berio

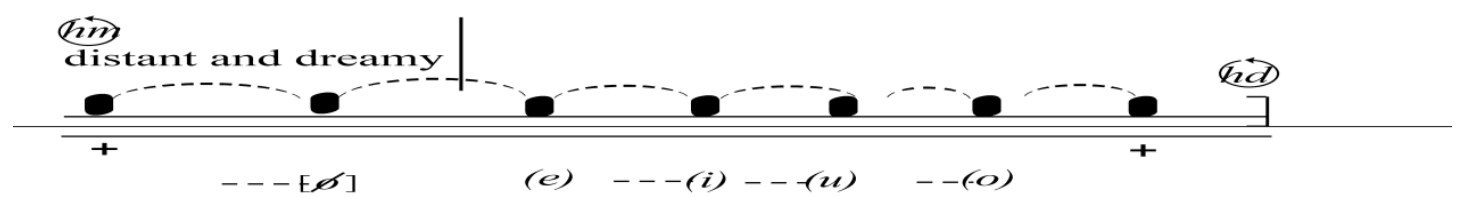

The representative works that expressing the musical effect feelings in lots of 'new voice' works include the Sequenza III for Voice written by Berio, which is the outstanding result of human voice technicalization, specialization and systematization, besides, it is unique and rises above the good and bad mixed modern music. He has adopted various sound effect methods of human voice and formed the unique style, besides, the presentation is not towering, on the contrary, along with performers' natural and excellent display, it has made the sound be more cohesive, fluent and harmony. Apart from that, the content presenting the works is more exquisite and colorful with an endless aftertaste, and its 'composite but not complicated', 'free but not chaotic' features and theatricality with much listening change and much feeling change are obvious.

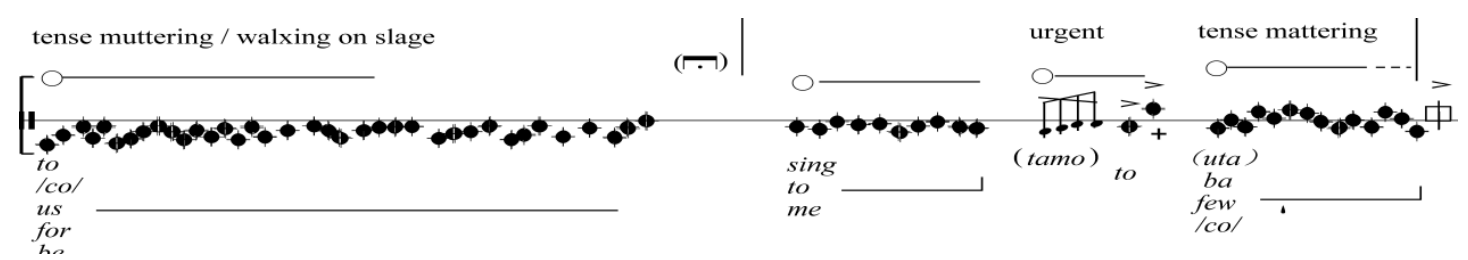

\section{Non-semantic Property of Lyrics}

Another feature of 'new voice' is the non-semantic property of lyrics. When the language and human voice as the resources, in most cases, the works lay emphasis on the sound effect of speech rate, pronunciation tone and human voice timbre. Many works borrow language and sound materials from the sister arts, such as modern literature, poetry, ancient poetry and various interjection, besides, the expression intention, form and state mostly stay in the whole emotion demand and expression spirit of the work so that to make the music manifest and conform to the freedom.

Example 1: Song of the Earth written by Ye Xiaogang mostly takes the traditional Chinese poetry of the Tang Dynasty as the lyrics and takes the chanting as the means, besides, for the expression method, it mostly adopts the natural pronunciation and general singing of character pronunciation and first character as the main expression method. Therefore, he has deviated from the expression frame of any traditional poetry of the Tang Dynasty and serve semantics and sound effect.

Music score example: Song of the Earth

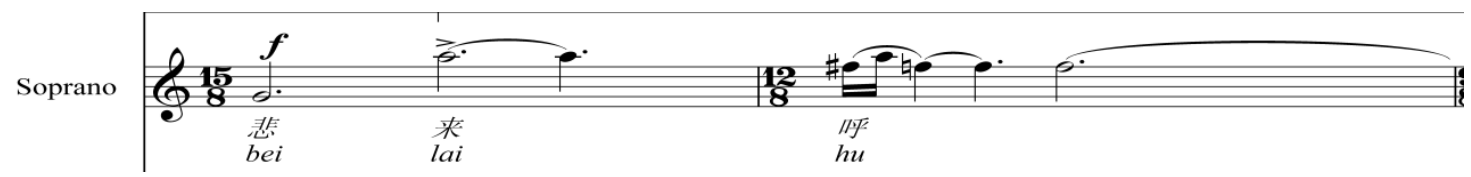

Another feature of 'new voice' works is the vocalization of lyrics. For the Italian bel canto, nothing is better than the vocalization cavity of pronunciation, which is the most essential and 
lasting, that is to say any character should be pronounced out with vibration smoothly through a cylindrical cavity and the finishing of that needs the help of Italian language vowels 'a,e,i,o,u' so that to get the bright and concentrated sound. This point has been borrowed and played sufficiently in modern 'new voice' works.

Example 2: For the art songs of LuoZhongrong, the people who have sung them know that the difficulties of the works are the timbre selection, style expression and lyrics interpretation, besides, the breath regulation and technical control are also the difficult points for singers to challenge. The author has taken elaborate design in musical lyrics writing and expression, for example, in order to have better resonance function through effectively expanding the oral cavity, the author has potentially emphasized the mouth-opened vowels 'a, e, o'

Music score example: Pick Lotus in the River

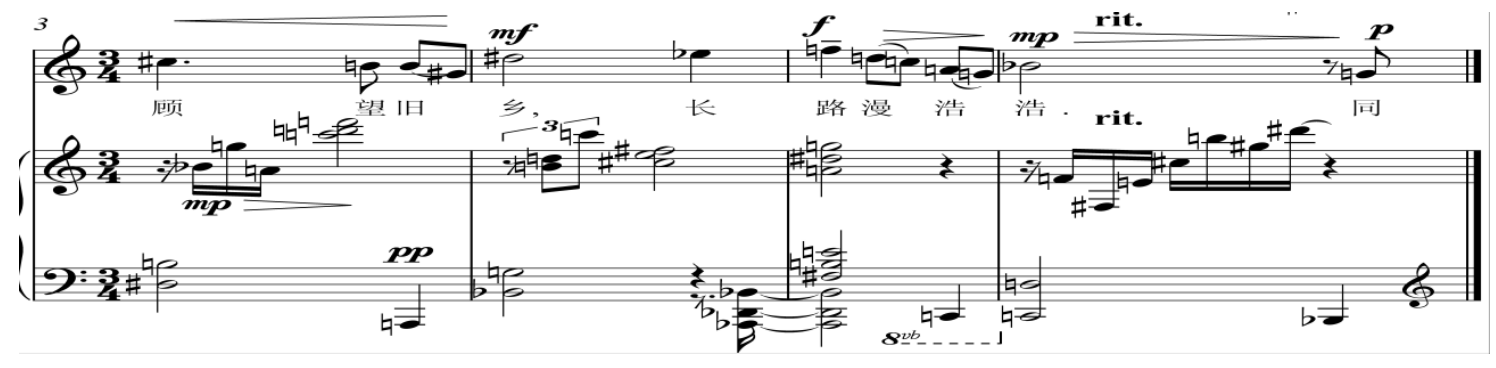

Example 3: the work Sequenza III for Voice written by Luciano Berio(1925-2003)

The author's another method for lyrics processing is representing one word with one monosyllable, such as gi stands for give, wo stands for woman, co stands for come etc. Obviously, except that the processed words by him have the technical meaning of composition, the lyrics no longer have the literal meaning.

Music score example: Sequenza III for Voice

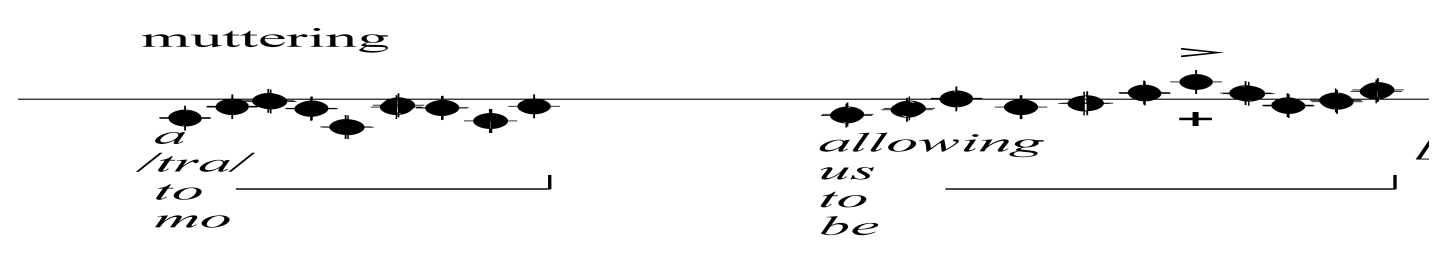

\section{Thought About 'Reorganization' and 'Innovation'}

The prosperous development of modern music has contributed to the emergence of various musical elements, for example, in the aspect of musical notation, the new musical notation method has appeared according to the need of works content, among which, there is electric notation, letter notation, table notation and exclusive notation signs for percussion instruments, apart from those, there is also the new notation, such as the unconventional notation for human voice singing and notation for other musical instruments etc. Sequenza III of Berio for Voice has the signs of one-line music score and three-line music score, which has provided accurate music score materials and made the singing performance mixed but not confused, compact but not crowded. For the Phantom of George Crumb, it has provided the music score with broad sound effect for piano and also displayed accurately and abundantly the content that the piano, as the sound of any part of musical instrument, serves the works. We can imagine that adding different notation methods into any 
modern 'new voice' musical works for the diversity of note and language expression and multidimensional uncertainty of emotion all can display the creative talents of the composers to great extent. From voiced to unvoiced, from ordered to disordered, from musical sound to sound effect, finally from scattered to changeable, besides, opening the spirit road from desert to oasis are all the important factors for the recreation of 'behavior music, concept music, environmental music, occasional music, mechanic music, performed music, abstract music, square music, stereo music, electric mixed music 2 'and the 'new voice' music of great reputation and disputation.

For the organic combination of various sounds, try to use different pronunciation styles, different singing ways, even different emotional sound effect in one work, besides, make use of multifaceted materials, which is colorful and involves various aspects. For example, China's new generation of excellent composer Ye Xiaogang and world composer Mahler are called 'master of silence' 'master of temperance', which is because that both of them have the work with a same name Song of the Earth; Difficult Raods in Shu and A Madman's Diary written by GuoWenjing; the art songs Loushan Pass and Poem on the Moon of Mount Emei with the Chinese poetry as the lyrics written by LuoZhongrong; opera Life Like the Strings and symphonic suite Mountains and Folk Custom; indoor fable opera Bet Your Life written by Wen Deqing. In these works, the authors have used the the unconventional human voice effect and proper content enriching the sound concept, which has embodied the integration property of musical multi-culture and expanded the musical expression strength, besides, these can manifest the Chinese 'new voice' concept works are experiencing changes and advancing along with new music development.

The innovation at any time is what the people expected and it may needs time to test, however, that can find one way out for people's inner hidden emotion to release and render all is the new experience. For the comparison between western and eastern music, China's music is relatively late for several decades in the road of pursuing new music, however, from the 1980th to today, the development of new music is becoming more and more fierce and it almost covers the entire thought of contemporary musicians and nowadays what we have seen is the bringing forth the new through the old, alienation and analyzing mentally, breaking the routine, abandoning traditional writing ways and thought and opening up the boundaries-breaking new type idea. The emergence of 'new voice' and the fission of modern musical context have made us feel that for the musical development, the glorious and magnificent cultural progress of human's history has reached the top of humanization, which is the spirit worship, self-enhancement of perceptual life and completeness of rationalism speculations, besides, it affirms the legality of human's central place and aesthetic creativity of subject's self-consciousness and freedom. No matter for the various human voice sound effect of Berio'sSequenza III for Voice, or the voice from the bottom of the heart in George Crumb's Phantom, or the combination of LuoZhongrong'stranditional poetry with new composing ways, or the combination of Chen Qigang's traditional bel canto soprano with line-cavity of traditional Peking opera Qingyi(female role) or the musical new experience etc. brought by the more and more 'poem style' and 'Chinese style' appeared in pop music are all the echo, rendering, twittering and whoop and they are all strengthening 'tradition+innovation=new music concept' + 'nature+alienation=breakthrough of new sound effect' =definition of 'new voice'.

\section{Conclusion}

'Foreign things serving China' 'view the world by standing on the shoulder of giant' may be the traditional method most worthy of borrowing, which just like the product of multi-thinking and the 'mosaic' concept of post-modern music is also the demonstration of multi-culture development, which market prospect cannot be ignored and it inevitably will be the innovation, breakthrough, 
fusion of the new music, besides, will go forward along with the musical wave and be the best result of globalization. Just like that, we should taste and think of the well-knowing saying of modern musician master Milton Babbitt that 'who cares about whether you listen to or not', and maybe he himself also is just sticking to the 'future' persistently. Maybe unknowingly, we have pushed the culture of the whole era forward.

\section{Bibliography}

[1]XuChangjun.Sequenza III for Voice written by Berio.MusicComposition.2003(2).

[2]Luqi.The New Human Voice Under the Background of Chinese New Music[J].People’s Music, 2007.

[3]Li Xi’an.Dialogues of Modern Music Thoughts, Beijing, People’s Music 1986(2).

[4]Li Shiyuan.One New Concept 'New Music’, Wuhan, Brass Bells, 1999(2).

[5]Dai Dingcheng.Various Chords Structure of Pentatonicism Connotation of LuoZhongrong Vocal Music Divertimento, Shanghai, Music Art, 1994(2).

[6]Liwan. Human Voice Reform and Others[J].Reading, 1995(10).

[7]Liu Juanjuan.Processing Methods for the Human Voice Melody of LuoZhongrong Art Songs[J], People’s Music, 2006

[8][America]Peters Hansen.Introduction to Twentieth Century Music[M].Beijing.People's Music Publishing House, 1986.the second volume.

[9]Tongxin.The Course Of Modern Notation[M].Changsha: Literature and Art Publishing House of Hunan, 2003.37.

[10]QianRenping.Chinese New Music.Shanghai Conservatory Of Music Publishing House, 2005 